CIUDAD Y TERRITORIO

ESTUDIOS TERRITORIALES

ISSN(P): 1133-4762; ISSN(E): 2659-3254

Vol. LII, № 204, verano 2020

Págs. 247-262

https://doi.org/10.37230/CyTET.2020.204.04

CC BY-NC 4.0

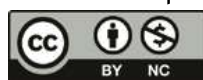

\title{
Integración urbanística de proyectos y planes portuarios en Las Palmas de Gran Canaria (1883-1991)
}

\author{
Antonio A. RAMÓN-OJEDA \\ Alejandro GonzÁlez-Morales ${ }^{1}$
}

Departamento de Geografía. Universidad de Las Palmas de Gran Canaria

RESUMEN: Dentro del proyecto Global South. Puertos y desarrollo económico y social en el Atlántico Meridional, investigamos las transformaciones inducidas mutuamente entre el puerto y la ciudad de Las Palmas de Gran Canaria. Estudiamos el crecimiento y expansión tanto del puerto como de la ciudad para intentar comprender cómo se articula el espacio de confluencia entre ambos. Nos planteamos la siguiente hipótesis: la relación entre la ciudad y su puerto ha evolucionado durante todos estos años, no sólo en el plano material o físico, sino incluso a nivel conceptual en cuanto a cómo son entendidos ambos espacios por sus respectivos planes y proyectos de construcción. Queremos saber si la transformación observada en el ámbito de confluencia del puerto y la ciudad obedece a la forma en que han sido concebidos ambos espacios. Para ello, consultamos los planes y proyectos que han definido el origen y desarrollo del puerto, así como los planeamientos de la ciudad desde 1898 hasta la actualidad. Hemos llevado a cabo un trabajo de análisis sobre la evolución del planeamiento urbanístico y portuario para determinar si se ha producido un cambio en el paradigma o forma de enfocar estos documentos, cómo se ha producido esta evolución y qué momentos han sido claves en dicho proceso.

PALABRAS CLAVE: Proyectos y planes; Espacio urbano-portuario; Diálogo puerto-ciudad; Paradigma.

Recibido: 14.01.2019; Revisado: 25.02.2019

Correo electrónico: toni.ramon@ulpgc.es No ORCID: https://orcid.org/0000-0002-4578-5185;

Correo electrónico: Alejandro.gonzalez@ulpgc.es No ORCID: http://orcid.org/0000-0002-2659-9678

Los autores agradecen los comentarios y sugerencias realizados por los evaluadores anónimos, que han contribuido a mejorar y enriquecer el manuscrito original.

${ }^{1}$ Este trabajo se enmarca dentro del proyecto de Global South. Puertos y desarrollo económico y social en el Atlántico Meridional (1850-1910). HAR2015-64044-R MINECO/FEDER, UE. 


\section{Territory, town and habitat in 2011 Malaga Urban Planning: farming and urban planning in Málaga Hills}

ABSTRACT: Inside the Global project South. Ports and economic and social development in the Southern Atlantic Ocean we investigate the mutually induced transformations between the port and the city of Las Palmas de Gran Canaria. We study the growth and expansion of both, the port and the city, to try to understand how the space of confluence between one and the other is articulated. We propose the following hypothesis: the relationship between the city and its port has evolved during all these years, not only in the material or physical plane, but also at a conceptual level as to how both spaces are understood by their respective plans and projects. We want to know if the transformation observed in the area of confluence of the port and the city is due to the way in which both spaces have been conceived. For this, we consult the plans and projects that have defined the origin and development of the port, as well as the planning of the city from 1898 to the present. We have carried out an analysis work on the evolution of urban and port planning to determine if there has been a change in the paradigm or way of focusing these documents, how this evolution has occurred and what moments have been key in that process.

KEYWORDS: Projects and plans; Urban-port space; Port-city dialogue; Paradigm.

\section{Introducción}

asta el siglo XIX el crecimiento de la ciudad de Las Palmas se vio limitado debido a las poco alentadoras condiciones fisiográficas, pero sobre todo económicas (MARTín, 2001). Sin embargo, la construcción del puerto a partir de 1883 generó un crecimiento muy importante de la capital (BurRiel, 1974, CÁceres, 1980), haciendo crecer el barrio de La Isleta ${ }^{2}$ hasta convertirlo en un polo de atracción para la ciudad histórica (FIG. 1), constreñida hasta ese entonces en los barrios de Vegueta y Triana. Desde ese momento comienza una historia de relación entre la ciudad y su puerto. Esta relación se mantiene mientras se produce la formación urbana de la ciudad baja, es decir, hasta aproximadamente la década de 1970; luego, ya consolidada esta parte de la ciudad, se entra en otra fase de transformación-adaptación a los intereses de ambos espacios.

De hecho, el presente estudio se cierra con el final del siglo XX porque desde hace aproximadamente 30 años la franja litoral colindante con el puerto no

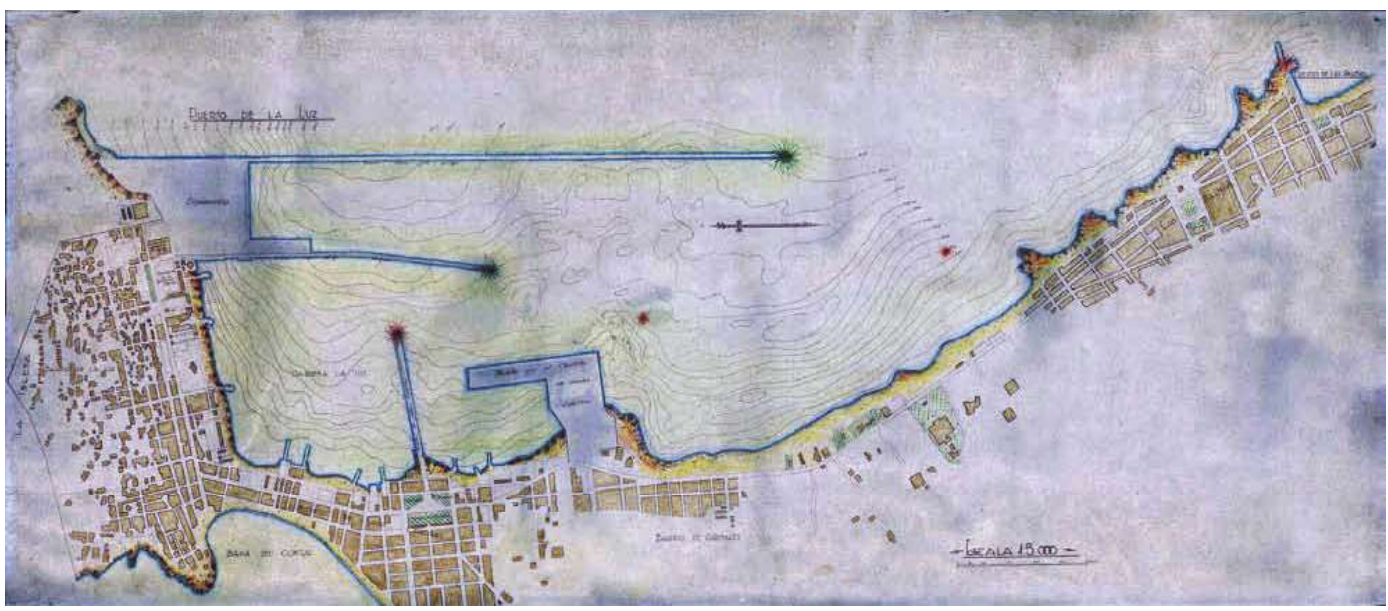

FIG. 1/ Mapa de Las Palmas de Gran Canaria y su puerto (1940)³.

Fuente: Archivo de la Autoridad Portuaria de Las Palmas.

2 Plan General de ampliación del Puerto de La Luz (1947) de Pedro Morales, Foto en anejo 3. Vista del barrio de La Isleta ya muy consolidado.

${ }^{3}$ Se aprecia el crecimiento de los barrios de Santa Catalina y La Isleta junto al puerto de La Luz. Al sur (derecha en la imagen), se ve dónde empieza la ciudad vieja (indicado como Puerto de Las Palmas). La carretera del puerto (hoy calles urbanas como León y Castillo, Albareda y Juan Rejón) sirvió como elemento que vertebró el crecimiento de la ciudad. 
ha experimentado transformaciones significativas en su estructura urbana. En tal sentido, se justifica que el periodo de estudio sea desde finales del siglo XIX (inicio de las obras portuarias) hasta la consolidación de la ciudad baja en contacto con el puerto, hecho que sucede en la década de 1970.

El presente trabajo es un análisis diacrónico de los proyectos y planes portuarios y urbanísticos de Las Palmas de Gran Canaria que se efectúa con el objetivo de entender el diálogo que se ha establecido entre ambos espacios desde la proyección técnica de los mismos. Se pretende analizar la evolución conjunta de ambas realidades estudiando para ello los planes y proyectos sobre los que se han gestado sus respectivos procesos de expansión. Partiendo de la hipótesis de que existe una influencia directa en el desarrollo del puerto y de la ciudad, nos interesa conocer cómo ha sido el proceso de integración entre ambos y especialmente, en el espacio de confluencia. En este sentido, se quiere comprobar si ha existido una evolución del rol que el puerto juega en relación al diseño y expansión de la trama urbana, cómo han evolucionado ambos y si desde el mismo proceso de planeamiento o diseño se ha producido una integración de estos espacios.

Planteamos que ha existido un cambio de paradigma en la producción del espacio de confluencia entre el puerto y la ciudad, llegando incluso a afectar a la forma en la que se interpreta la relación mutua entre ambos por parte del proyectista o del planificador. En otras palabras, analizamos cómo han sido "pensados" estos espacios, y si la ciudad y la interacción del puerto con su entorno se han adoptado como criterio de base en la planificación.

El puerto es una realidad geográfica que juega un papel fundamental en los transportes y la logística y, por tanto, su finalidad es sobre todo económica. En términos de la memoria del proyecto de "Nuevo muelle por el Castillo de Santa Catalina", el puerto es una "estación comercial"; pero al mismo tiempo constituye un polo de atracción para la expansión de la ciudad, llegando con el tiempo a conformar parte del paisaje urbano en el que acaba por integrarse. Cuando esto sucede, se genera un espacio tensionado (DELGADO, 1998), donde aparecen conflictos entre la trama urbana, como lugar de residencia, y el recinto portuario, que juega un papel esencialmente funcional. A medida que este último crece, se establecen en él actividades de naturaleza industrial que comienzan a resultar incompatibles con el desarrollo urbano, al que sin embargo y contradictoriamente atrae.

En efecto, durante buena parte de la historia del puerto, esta manifiesta incompatibilidad no fue ni un condicionante para su crecimiento, ni un aspecto valorado de forma especialmente negativa por la ciudadanía (SUÁREZ, 2005). De hecho, la ciudad creció hacia su puerto como si las actividades que en éste se desarrollaban no le afectasen o incomodasen. En este marco, la compatibilidad de usos no era uno de los criterios de planificación considerado por parte de los redactores de los distintos proyectos y planes sobre los que se expandió el puerto. Sin embargo, este enfoque fue variando con los años, de tal manera que, al comienzo prevalecía casi exclusivamente el criterio funcional a la hora de planificar las infraestructuras portuarias, pero con el tiempo la planificación se hizo bajo preceptos más globales, de manera que hoy en día existe una mayor integración entre la actividad portuaria y los intereses urbanos.

Es precisamente este proceso de transformación, física, pero también conceptual, el que analizamos en el presente trabajo. Para ello, hemos acudido a fuentes básicas como son: el archivo de la Autoridad Portuaria de Las Palmas, la Oficina de Planeamiento del Ayuntamiento de Las Palmas de Gran Canaria, Museo Canario, fondo cartográfico y fotografías aéreas del Departamento de Geografía, fototeca del Cabildo de Gran Canaria (a través de la IDE Canarias), fondo fotográfico de la FEDAC, archivo digital de hemeroteca de la aplicación Jable (ULPGC), Delegación de Hacienda y Archivo Histórico Provincial. Nos apoyamos en documentación cartográfica y fotográfica, complementada con documentos de planeamiento, correspondencia oficial, artículos de prensa, documentos de tramitación, y otros a los que hemos tenido acceso en los anteriores organismos.

Por supuesto, la consulta de los diferentes proyectos y planes de desarrollo, especialmente del puerto, pero también de la ciudad, han sido fundamentales para la realización del presente estudio.

Asimismo, también resultan de interés publicaciones de investigadores como MARTíN GALÁN (2001 y 2007); HeRrera Piqué (1978 y 2008); CÁCERES MoRAles (1987); Holm (2013), entre otros.

El trabajo se presenta dividido en cinco apartados: el primero es una introducción que pretende definir el objeto y ámbito de estudio, así como nuestro planteamiento, hipótesis y también la metodología y fuentes empleadas. En un segundo apartado se realiza una aproximación al marco geográfico e histórico para poner al lector en antecedentes. Asimismo, también hemos incluido una breve relación y valoración de las principales fuentes empleadas en el estudio: los proyectos y planes del puerto de La luz y Las Palmas. 
Finalmente, el apartado cuarto constituye el corpus central, con el que repasamos cómo ha evolucionado la relación puerto-ciudad, según un proceso que hemos dividido en tres etapas: una primera etapa "proyectista"; una segunda de planificación esencialmente económica de base industrial y una tercera urbanística o post-industrial, donde se incorpora el puerto como espacio de ocio y esparcimiento de la ciudad en el marco de una economía terciarizada. Finalmente, exponemos unas conclusiones extraídas a partir de la elaboración y desarrollo de la investigación.

\section{La planificación del puerto y la ciudad}

La Autoridad Portuaria de Las Palmas mantiene en sus archivos los diferentes proyectos y planes que han servido para los desarrollos portuarios. Estos constituyen una fuente documental esencial en el presente estudio y son los que a continuación se relacionan ordenados cronológicamente y referidos de manera abreviada por el nombre de sus redactores (FIG. 2) ${ }^{4}$ :

El primer proyecto para el puerto de La Isleta fue obra de Clavijo y Pló, pero nunca se concretó. Sí lo hizo el de Juan León y Castillo, artífice del puerto de refugio (1881), para el que contó con la opinión de los marineros y hombres de la mar, a los que reunió para comentar su propuesta (CIRILO, 1947: 51).
En 1910, el Ingeniero Jefe de Puertos Eugenio Suárez Galván se encargó de redactar el Plan de mejoras del Puerto de La Luz. Pero intervino el Ramo de Guerra y se creó una comisión mixta, resultado de lo cual se encargó al Comandante Carreras Irigorri que presentase una propuesta acorde a los intereses del Ministerio de Marina. Ante dicha comisión se presentaron tres alternativas, la de Suárez Galván, la de Carreras Irigorri y un plan consensuado, resultando elegido este último. Se redactó bajo criterios económicos y militares.

En 1916 el ingeniero Jaime Ramonell Obrador redactó un Plan de servicios del Puerto sobre base de la comisión mixta de los Ministerios de Marina y Fomento ya comentada. Ramonell asumió las propuestas precedentes, a las que añadió, tal como se le había encomendado, un proyecto de carretera para unir el puerto con la ciudad. Este elemento será esencial en el desarrollo urbano. Hoy día constituye las calles León y Castillo, Albareda y Juan Rejón.

Entre 1924 y 1927 se redactaron los proyectos concernientes al relleno del por entonces muelle Primo de Rivera y dique de la COPPA. Participan en él tres ingenieros: Acedo, Artiles y Rodríguez Rodas. Este último presenta también una propuesta de urbanización para el barrio de La Isleta (Memoria del Puerto de La Luz, 1928) (FIG. 3). Nunca llegó a materializarse.

\begin{tabular}{ll}
\hline 1881 & Proyecto de Juan de León y Castillo (copia en el Museo Canario) \\
\hline 1910 & Plan de Mejoras del Puerto de La Luz (Suárez Galván) \\
\hline 1913 & Modificado del proyecto por Suárez Galván y Carreras Irigorri \\
\hline 1916 & Plan General de Servicios del Puerto de La Luz (Ramonell Obrador) \\
\hline $1924-1927$ & Proyecto de rellenos del muelle General Primo de Rivera de Acedo, Artiles y Rodríguez Rodas \\
\hline 1937 & Plan de obras en el Puerto de La Luz o Plan Conesa \\
\hline 1947 & Plan General de Ampliación del Puerto de La Luz (Morales) \\
\hline 1954 & Plan de obras, instalaciones, material y medios auxiliares de La Luz o Plan Picó \\
\hline $1960-62$ & Plan General de Ordenación del Puerto de La Luz de Granda (y Plan Canarias) \\
\hline 1964 & Plan General de Ordenación y Obras de los Puertos de La Luz y Las Palmas y anteproyecto \\
\hline 1971 & de la Dársena Exterior-Primera fase o Plan Vigueras \\
\hline 1982 & Plan de las obras de infraestructura del puerto o Plan Argenti Ulloa \\
\hline 1991 & Plan General de Actuación de Fernando Navarro Miñón \\
\hline
\end{tabular}

FIG. 2/ Relación de planes y proyectos portuarios de carácter general (1881-1991) ${ }^{\text {. }}$

${ }^{4}$ Normalmente aparecen firmados por el Ingeniero Director del Puerto. 5 Se ha establecido esta fecha por ser un momento en el que la ciudad baja, en contacto con el puerto, se encuentra definitivamente consolidada. En cualquier caso, los documentos recientes pueden ser consultados a través de la web:

http://www.palmasport.es/. 


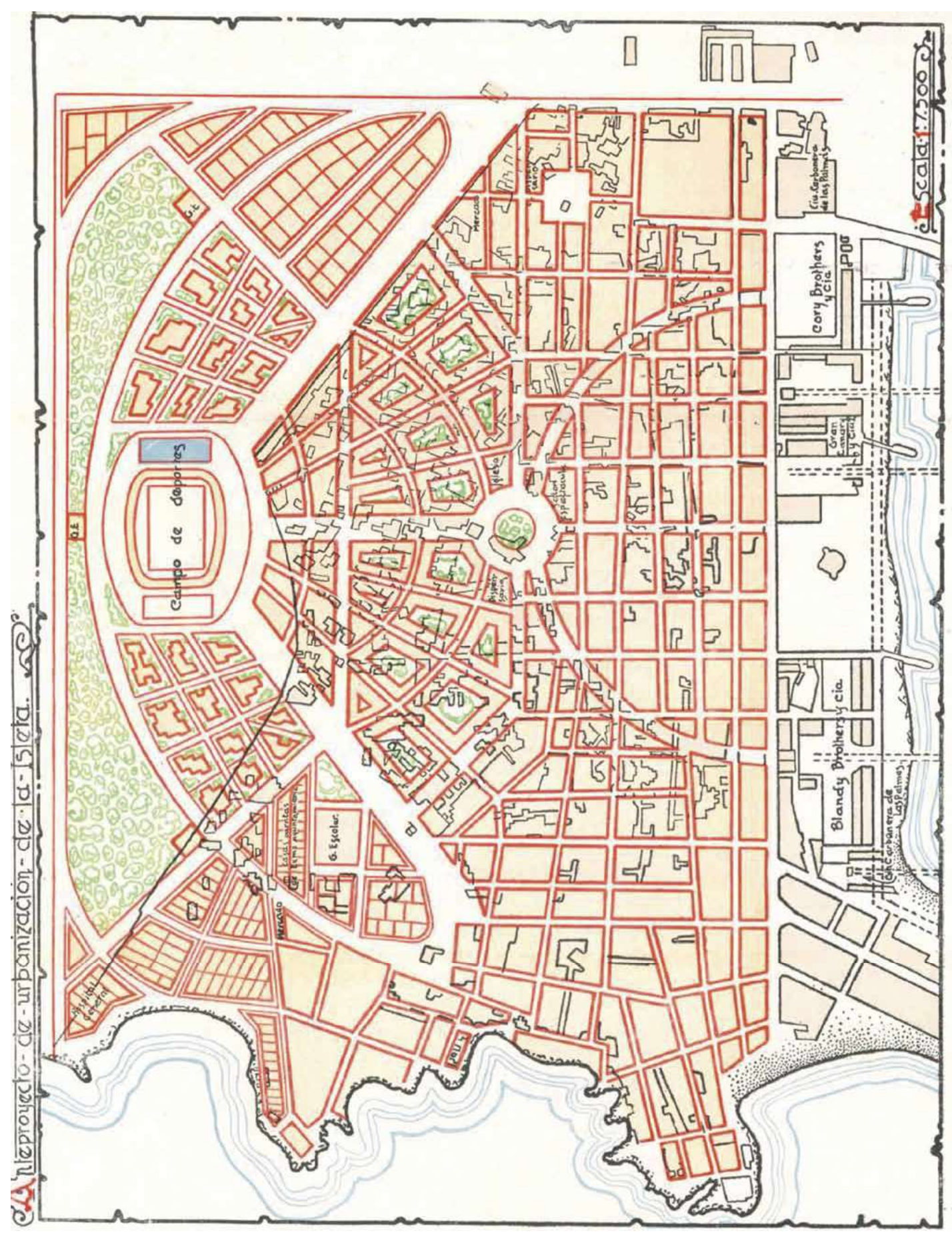




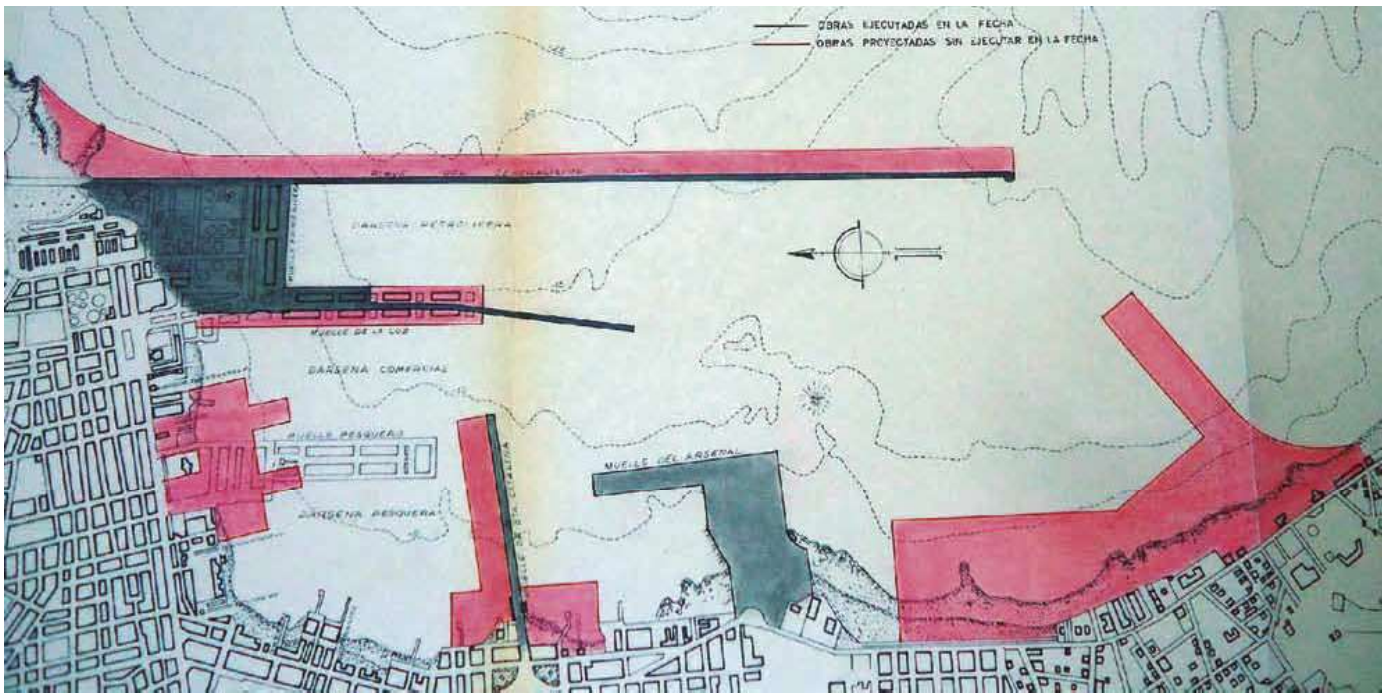

FIG. 4/ Propuesta de Pedro Morales Plequenzuelo para el Plan General de Ampliación del Puerto de La Luz (1947).

Fuente: Memoria del Plan Modesto Vigueras (1964). Archivo de la Autoridad Portuaria de Las Palmas.

En 1932 Antonio Artiles redactó el proyecto del muelle frutero (1937), que sería rápidamente incautado y convertido en base naval (1940). A día de hoy representa una de las reclamaciones más insistentemente solicitada por la ciudad.

En 1937 se aprueba el Plan de obras en el Puerto de La Luz de Pérez Conesa, si bien éste tiene nula incidencia sobre la ciudad, pues planteaba únicamente el ensanche del Muelle de La Luz, sin que la actuación tuviera efectos sobre la trama urbana.

Lo contrario puede decirse del Plan General de Ampliación de Pedro Morales (1947), que comprendía no sólo obras estrictamente portuarias, entre ellas el ensanche del muelle de Santa Catalina y los muelles de ribera contiguos, sino también obras de urbanización y edificaciones y servicios, aunque todos destinados a actividades propias del puerto. Morales planteó una red de alcantarillado, edificio para la Junta, e incluso la urbanización de terrenos en La Isleta. Además, también planteó una explanada y dique que habría supuesto la transformación de la franja litoral comprendida entre la playa de Las Alcaravaneras y el Barranquillo don Zoilo (calle Juan XXIII) (FIG. 4).

El Plan de 1954 de Rafael Picó estaba centrado en el litoral ubicado entre Santa Catalina y el

\footnotetext{
${ }^{6}$ Informe relativo a las distribuciones posibles de servicios en el Puerto de la Luz. Caballero de Rodas. 1940.

${ }^{7} \mathrm{El}$ antes referido informe de Caballero de Rodas (1940) llegó a proponer la construcción de un muelle de ribera entre el arranque norte de la base naval y el muelle Santa
}

muelle pesquero. Este último era una prioridad ante los nuevos acontecimientos internacionales y el incremento de la actividad pesquera que venía produciéndose ya desde finales de los años 30 , repuntando luego al finalizar los conflictos bélicos (Plan Conesa, 1937; Informe del Ingeniero Director Caballero de Rodas ${ }^{6}$, 1939).

El comienzo de la década de 1960 es especialmente importante, como luego tendremos ocasión de desarrollar más detenidamente. De una parte, se redacta un proyecto de ampliación del puerto, casi un esbozo, dentro del marco del Plan de Estabilización Económica o Plan Ullastres. Por otra parte, y casi al mismo tiempo, Granda Villar, como Ingeniero Jefe, redacta el Plan General de 1962, encargado por la Superioridad. Se centró principalmente en dar soluciones a las necesidades más perentorias, y en consecuencia planteaba un gran muelle pesquero, que iría localizado próximo a la zona del mercado del puerto y por tanto en la confluencia con la trama urbana ${ }^{7}$. La propuesta era claramente económica y olvidaba considerar el entorno urbano de su emplazamiento, con el que entraba en conflicto.

Tras el rechazo que supuso el plan de 1962 por múltiples y diferentes agentes sociales, el recién

Catalina para la flota pesquera, e incluso habilitar el Muelle Virgen del Pino para esta actividad. Pero este proyecto chocó con los intereses del Ministerio de Aviación y su muelle de hidroaviones, por lo que no llegó a materializarse. 
nombrado Ingeniero del Puerto, Modesto Vigueras, fue el encargado de diseñar un nuevo plan que contemplara la propuesta de ampliación del dique exterior, así como un emplazamiento menos molesto para las actividades pesqueras. Este documento fue redactado en un tiempo record, pues Vigueras es nombrado en septiembre de 1963 el plan sale a información pública según anuncio en el BOP de 23 de marzo de 1964 y se aprueba en julio de ese mismo año.

Los siguientes planes son de escasa entidad y corresponden a 1971 (Argenti Ulloa) y 1982 (Navarro Miñón). Coinciden justo con el momento de transición que marca la Ley del Suelo de 197576 , que como más adelante se verá, resultará trascendental en la nueva concepción del planeamiento al adoptar una visión más integral. Los planes sucesivos corresponden a una época en que la Ciudad Baja está perfectamente definida y acotada, y apenas hay crecimiento urbano en el ámbito que nos ocupa, pero sí una transformación del mismo que obedece a su adaptación a los nuevos intereses urbano-portuarios, especialmente en el entorno del parque Santa Catalina, la base actual de cruceros.

Durante este período de 100 años, en los que se construye la trama urbana junto al área portuaria, la ciudad redactó seis planes que pueden ser calificados como de "generales" para el conjunto de la urbe: En 1898, el de Laureano Arroyo; en 1911 el de Fernando Navarro; en 1922, el firmado por Miguel Martín-Fernández y en 1944 el de Secundino Zuazo, posteriormente modificado por Antonio Cardona, quien casi rehízo por completo la propuesta de Zuazo (MiRAlLAVE, 1990) redactando un nuevo plan, el quinto, aprobado en 1952. En 1962 Guerrero Aroca y Sánchez de León firman un Plan General con el que se acaba por consolidar la ciudad baja. Aunque en 1972 el Ayuntamiento aprobó el avance de un nuevo Plan, éste sería pospuesto hasta 1978 debido a la aprobación de la Ley del Suelo de 1975. Su incidencia sobre el espacio urbano-portuario sería mínima al haberse producido ya la colmatación del mismo.

Por tanto, el de 1970 será un decenio clave, más en el cambio de paradigma en la forma de entender la relación puerto-ciudad, que en la producción urbana, que como hemos dicho estaba prácticamente definida por ese entonces.

En este sentido, el Plan Especial de la zona de servicio del Puerto de La Luz, redactado por José $M^{a}$ Hernández León y aprobado en 1991, confirma el nuevo enfoque planificador. Es el primero de su naturaleza que contempla expresamente la relación puerto-ciudad, lo hace en un apartado homónimo, el 2.2.4 de la memoria, en el que se considera, eso sí, escuetamente, el PGOU de la ciudad y las infraestructuras viarias.

Desde este momento los planes portuarios y urbanos tendrán una mayor integración. La década de 1980, pero sobre todo la que arranca con este plan de 1991, marcan ya el período de transformación del espacio urbano-portuario, que se irá adaptando cada vez más a sus nuevas funciones, relacionadas con una economía terciarizada. Desde este momento se inicia un nuevo diálogo entre ambas realidades resultado del cuál es la transformación del litoral capitalino en su contacto con el puerto. En esta etapa no hay nuevo crecimiento urbano litoral, pero sí estrategias orientadas a alcanzar una mayor relación entre los espacios urbanos y portuarios (DEL NERO, 2007 y 2008). El puerto ahora crece hacia la península del Nido, alejándose de la ciudad y permitiendo nuevos proyectos urbanos en la zona del istmo (GONZÁLEZ \& ARMENGOL, 2006), especialmente en el entorno del muelle de Santa Catalina, hoy base de cruceros: rehabilitación de los viejos almacenes portuarios de Elder y Miller, construcción del intercambiador del transporte urbano y el Parque Islas Canarias, nuevo centro comercial El Muelle o el más reciente acuario Poema del Mar, entre otras intervenciones orientadas todas ellas a crear espacios de ocio muy vinculados con el turismo de cruceros.

\section{La evolución "urbanística" de los proyectos y planes portuarios}

Mediante el análisis de los diferentes planes y proyectos redactados en relación a las obras del puerto de La Luz es posible estudiar el cambio de paradigma y enfoque, incluso de objetivos esenciales que cada uno de estos documentos técnicos emplea como base estratégica para exponer sus planteamientos. Parece claro que existe una etapa que, siguiendo la nomenclatura de la Ley del Suelo de 1976, podemos definir como socio-económica, por ser éstos los criterios fundamentales a la hora de abordar tanto proyectos, como planes. Existe luego una segunda etapa más integradora, que según el mismo texto debería ser referida como de "planeamiento físico", pero que preferimos denominar como "urbanística", por existir precisamente una ordenación urbanística del territorio, en términos de la propia Ley de 1976.

Pero también observamos una evolución en la forma de enfocar los planes de crecimiento del puerto que nos lleva a diferenciar dos subetapas dentro de la fase que hemos denominado socioeconómica, de manera que finalmente podemos establecer la siguiente periodización. 


\subsection{La etapa "proyectista". 1881-1937}

A lo largo de esta fase observamos la existencia de una cierta planificación, pero la transformación territorial se ejerce principalmente desde la redacción y diseño de proyectos. Éstos, son documentos esencialmente de ingeniería fundamentados casi en exclusiva sobre las estadísticas de tráfico de buques, mercancías y pasajeros como justificación de las obras de mejora y crecimiento del puerto. Son, por tanto, criterios claramente socioeconómicos los que imperan a la hora de diseñar los crecimientos portuarios.

Entre los documentos de planificación de esta etapa estarían los referidos de León y Castillo (1881); Los planes de Suárez Galván (1910 y 1913, este segundo en colaboración con Carreras Irigorri); el Plan Ramonell (1916) y los proyetos y planes de la tríada Acedo, Artiles y Rodríguez Rodas (1927).

Es interesante observar cómo los proyectos conjuntos de Acedo, Artiles y Rodríguez Rodas hacían especial hincapié en la solicitud de "un paseo para coches, tan necesario en la ciudad y jardines que proporcionen al público comodidad y acaben de alegrar el ambiente agradable de los puertos con su intensivo trabajo encuadrado por el mar". Asimismo, proponen un paseo marítimo al sur del castillo de Santa Catalina, que estaba ubicado donde hoy se encuentra el arranque de la base naval. Esta propuesta, junto con la urbanización de la explanada de Santa Catalina, también de estos autores, sería de las pocas iniciativas que desde planes o proyectos del puerto planteaban en esta etapa el diseño de elementos propios para la ciudad. Ni siquiera la vía de servicio proyectada con antelación (R.O. de 20-1-1854) era un elemento urbano, aunque al final acabara traspasándose a la municipalidad.

Respecto a la carretera del Puerto, cabe señalar que el proyecto de Ramonell para la construcción de esta vía es recogido por Miguel MartínFernández en el plano que realizara para el Plan General Urbano en 1922, y que la vía acabaría por convertirse en un elemento clave en la vertebración de la ciudad baja, lo que constituye un hecho sobradamente conocido (MARTín, 2001).

Urbanísticamente, a esta etapa corresponden los planes de Arroyo (1898), Fernando Navarro (1911) y Miguel Martín (1922), que conceptualmente resultan muy diferentes. El primero responde a los cánones clásicos del ensanche. Arroyo diseña dos "ciudades": la zona vieja, que corresponde con Las Palmas, al sur y la nueva ciudad portuaria, al norte.

La parte vieja queda articulada en torno a los tres ejes viarios principales paralelos a la costa: Triana, Cano-Viera y Clavijo y San Francisco-Pérez Galdós, calles que tendrán prolongación hacia el norte por León y Castillo, Perojo y Paseo de Tomás Morales, respectivamente. En este sector la interacción de la trama urbana con el puerto es prácticamente inexistente. Por el contrario, al norte Arroyo planifica una nueva trama urbana, parcialmente sustentada en el eje que marca el dique transversal del muelle de Santa Catalina. Esta nueva ciudad queda trazada en tres unidades, de las cuales, la central (barrio de Santa Catalina) se configura en torno a un nuevo viario cuya calle principal es prolongación del muelle (actual calle Ripoche). Partiendo de éste, Arroyo traza una alameda en dirección este-oeste que finalizaría en una iglesia en el extremo opuesto al propio muelle (nunca construida). Esta calle vertebra la retícula tanto al norte, como al sur, definiendo por tanto la trama urbana de este sector de la ciudad.

Pero incluso en la "ciudad portuaria" existen dos ámbitos diferenciados. En el istmo y hasta los arenales de Santa Catalina, se proyecta una trama reticular ordenada ortogonalmente. En cambio, en La Isleta, la proliferación de chozas que son el resultado del asentamiento espontáneo y rápido que produjo la llegada de población atraída por las obras del puerto (MedinA, 1996) supuso un problema finalmente no resuelto y que derivó en una trama irregular que aún hoy día se mantiene. Además, Arroyo pasa por alto el espacio existente entre ambos núcleos, el puerto y la ciudad vieja, consolidando de esta manera dos núcleos diferenciados.

Fernando Navarro tiene una visión del todo continuista y apenas aporta novedad alguna sobre lo diseñado por Arroyo, al menos en su Plan General, en el que mantiene las mismas directrices de su predecesor. En cambio, en un proyecto suyo de 1914 para la ampliación del Parque San Telmo, el por entonces arquitecto municipal ya advierte que

"la Ciudad de Las Palmas es, y deberá ser en lo futuro, esencialmente marítima, desarrollándose por las playas hasta formar un solo núcleo con su puerto".

Esta frase queda consignada en la memoria del proyecto, bajo el epígrafe "Preliminares"

\footnotetext{
${ }^{8}$ Ocupación de terrenos ganados al mar para ensanche del parque San Telmo. Peticionario: Ayuntamiento de Las Palmas. concesiones administrativas, caja 66 - Legajo 61
} 


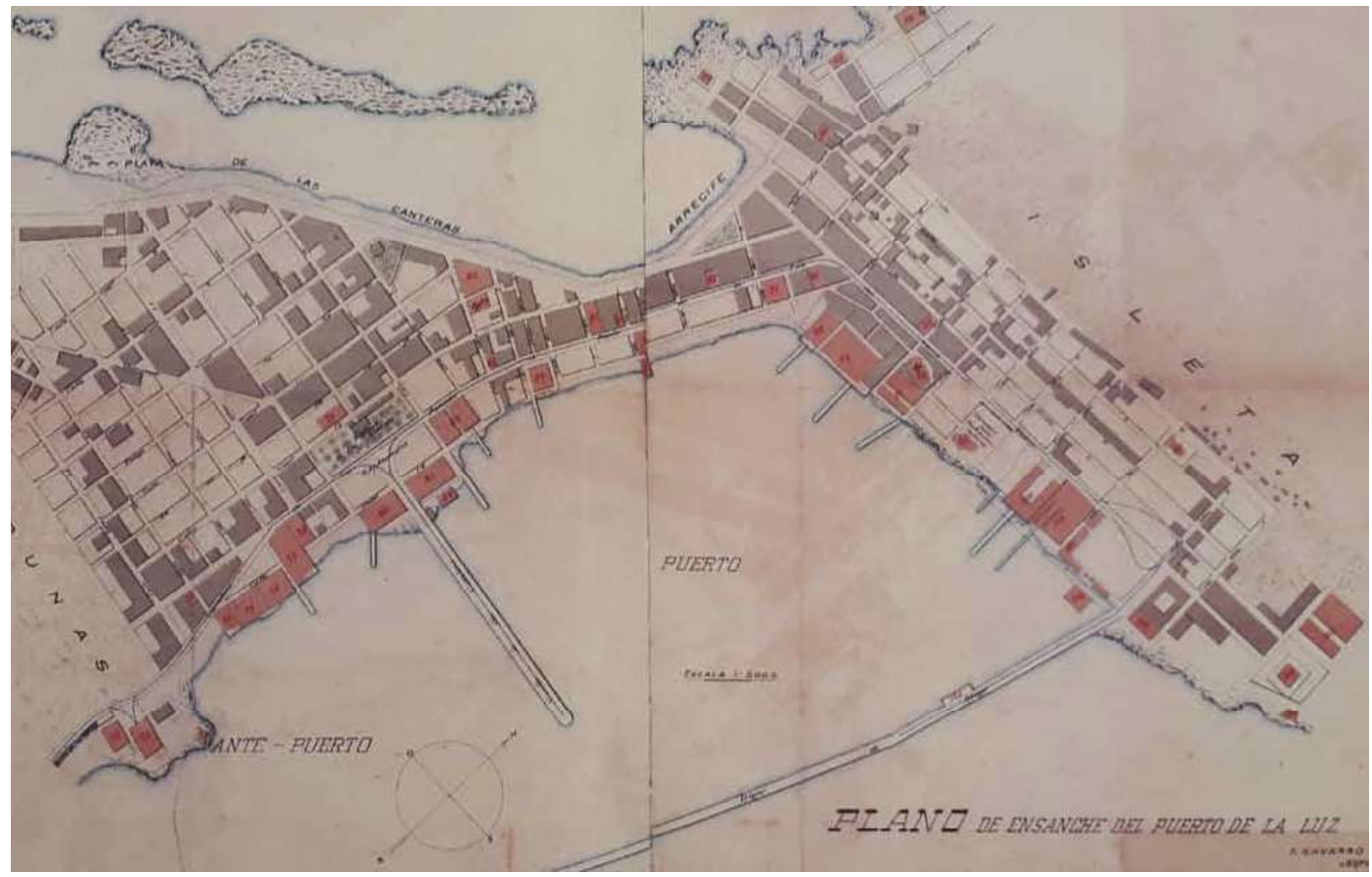

FIG. 5/ Plano de Fernando Navarro donde ordena los barrios de Santa Catalina y La Isleta.

Se pueden observar construcciones ya edificadas y también las manzanas planificadas.

Fuente: Tous \& HERRERA, 1995

Por otra parte, el planteamiento de Miguel Martín (1922) se centra sobre todo en el espacio vacío que habían dejado Arroyo y Navarro, esto es, sobre los barrios de Arenales-Alcaravaneras. Para Mesa y López diseña un eje paralelo al reticulado propuesto por Arroyo y el barrio se iría consolidando en las tres siguientes décadas según el damero planificado, mientras que en el sector intermedio entre "ambas ciudades", Martín diseña una ciudad jardín sobre lo que era el barrio de los hoteles, intentando seguir los esquemas orgánicos imperantes en la época y dictados por Ebenezer Howard. La trama del barrio de Mesa y López, al seguir la retícula en damero definida según el eje del muelle Santa Catalina, mantiene una vinculación directa con el puerto. Además, la avenida principal homónima es paralela a la calle Ripoche y su arranque se produce en la base del muelle frutero Virgen del Pino, que se construyó en 1937, y sobre el viario que durante las obras se utilizó para el transporte de arena. De hecho, y aunque el proyecto del puerto es de 1934 y por tanto posterior al planeamiento de Martín, sin embargo, éste en su planificación ya dibuja un dique justo donde luego se construiría efectivamente la actual base naval. Sobre este dique vertebra Martín la totalidad del barrio.
Por el contrario, la Ciudad Jardín manifiesta escasa interacción con el litoral y, por extensión, con el puerto, cuyas obras prácticamente finalizaban en el anteriormente referido muelle frutero (hoy base naval), mientras que el barrio de Ciudad Jardín comienza justo al sur de este último. El diseño aquí es orgánico, predominando las viviendas unifamiliares en parcelas independientes y manzanas que, aunque geométricas, rompen la linealidad que se observa en el barrio de Santa Catalina-Mesa y López. Miguel Martín se centra especialmente en el espacio de expansión urbana, casi no dice nada sobre la ciudad antigua, esto es Vegueta-Triana, y ni tan siquiera hace alusión a La Isleta, que no toma en consideración en su plan.

Durante esta misma etapa es significativo el ordenamiento del barrio de Santa Catalina que se lleva a cabo por parte de Fernando Navarro en su Plan de 1911 (FIG. 5), que juntamente con la propuesta de Rodríguez Rodas para el barrio de La Isleta (FIG 2), constituyen dos formas integradoras de entender el crecimiento urbano. En ambos casos la planificación se adopta en consideración de las infraestructuras portuarias, que sirven como elementos vertebradores de las barriadas. 


\subsection{La etapa "de planeamiento económico”. 1937-1975}

Comprende esta etapa los planes de Pedro Morales (1947); Rafael Picó (1954); Granda Villar (1960-62), incluye la propuesta del Plan Canarias y el no aprobado PGO, ambos supervisados por el propio Granda Villar); Modesto Vigueras (1964) y Juan Argenti (1971).

Un hecho novedoso tuvo lugar con el Plan de 1947 y es que incorpora por primera vez entre su documentación el Plan General Urbano (el de Zuazo, de 1944) e incluso asume en su propuesta la realizada por el arquitecto vasco de ampliar la ciudad hacia el este ganando terrenos al mar. De ahí la enorme explanada que Picó proyecta frente a la playa de Lugo, al sur de la de Las Alcaravaneras, y cuyo "ideólogo" había sido precisamente Secundino Zuazo. Éste sería el germen de un interesantísimo proceso, el de ampliación de terrenos urbanos hacia el este, que junto a la construcción de la avenida marítima, constituyen sin duda los principales hitos en cuanto al espacio de confluencia urbano-portuario ganados al mar. El proceso está perfectamente documentado en el Archivo del Puerto de Las Palmas.

Además, el plan de Morales también asumía reivindicaciones urbanas al proyectar los muelles de ribera adentrándolos hacia el mar más de lo estrictamente preciso para los fines portuarios. La ciudad ganaría mediante concesión una superficie próxima a los $50.000 \mathrm{~m}^{2}$, con casi tres kilómetros de litoral.

Pero ante este avance en el diálogo de ambas realidades geográficas, la ciudad y el puerto, que supuso el plan de 1947, sobrevino un aspecto económico y estrictamente portuario. El incremento de la actividad pesquera exigía una rápida solución ante la cada vez mayor demanda de infraestructuras destinadas a este fin. Esta situación generó mucha controversia entre los intereses portuarios (y pesqueros) y la propia ciudad. El plan Picó de 1954 (Anteproyecto General del Puerto Pesquero. FIG. 6) realizó un extenso estudio para lograr un puerto pesquero y en su largo catálogo de 15 alternativas se barajaron posibilidades de todo tipo, desde las que proponían un emplazamiento dentro de las dársenas existentes, hasta propuestas más descabelladas que llevaban el futuro muelle pesquero a La Isleta, o incluso a la playa de Las Canteras. Lo más interesante de este plan era que preveía la ampliación del puerto en una segunda etapa, ensanchando el dique del Generalísimo que se prolongaba hacia el sur $500 \mathrm{~m}$, y proponiendo construir un gran muelle-dique que arrancaba desde el muelle Las Palmas como contradique al puerto, además de

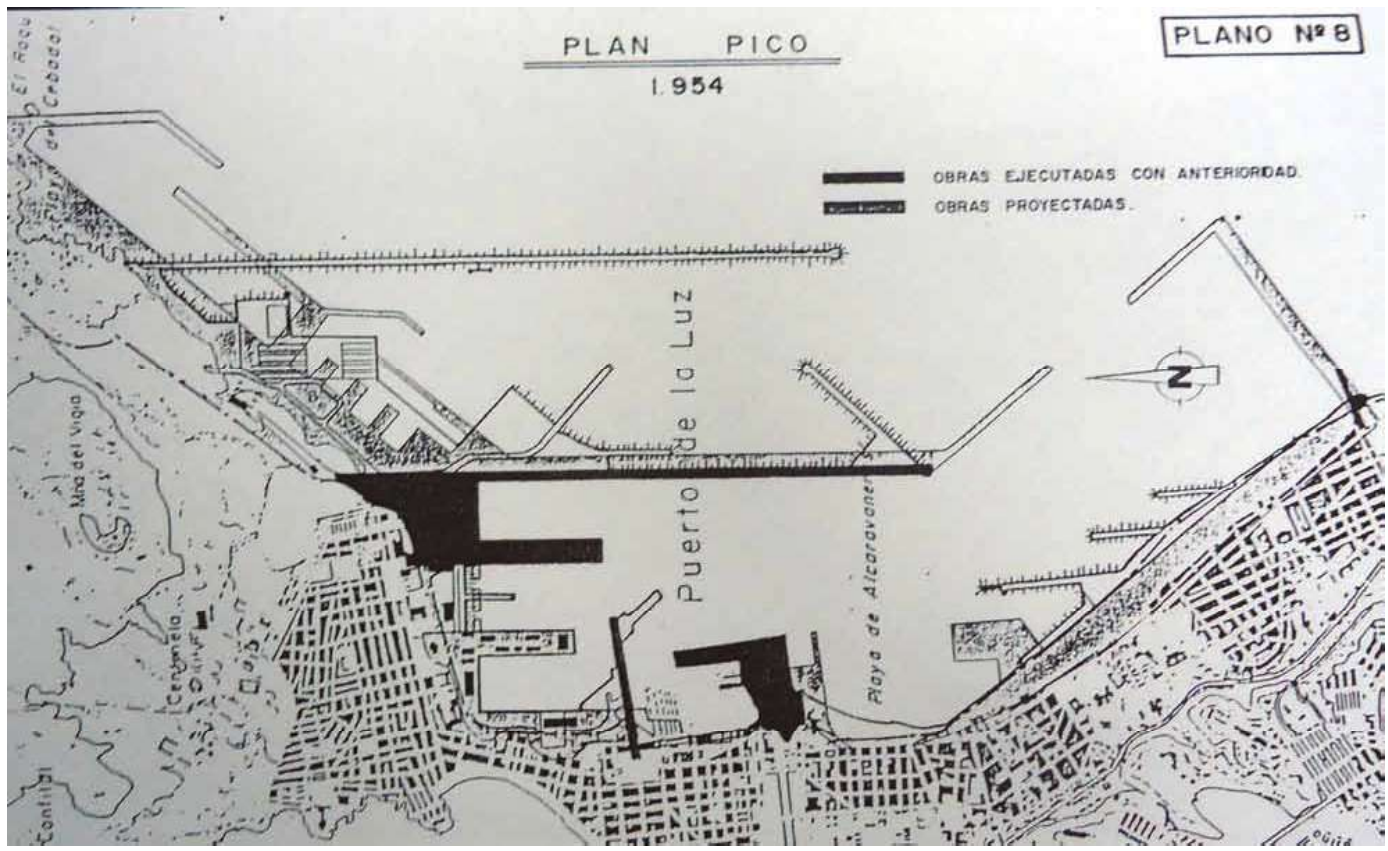

FIG. 6/ Esquema de la propuesta de Rafael Picó (1954).

Fuente: Memoria del PG de 1991. Archivo de la Autoridad Portuaria de Las Palmas. 
dos dársenas exteriores pegadas a la Isleta, donde irían las actividades petrolíferas y pesqueras. Era el de Picó un plan de envergadura, que representó una verdadera encrucijada en el devenir de la relación puerto-ciudad. Por una parte, el ingeniero proponía un ensanche del puerto creciendo hacia naciente, lo que suponía ampliar su actividad hacia el este, alejándose de la ciudad. Por otra, Picó proponía una obra de grandes dimensiones como prolongación del viejo muelle Las Palmas en Triana, lo que supondría de facto hipotecar por completo todo el frente litoral de la ciudad entre este punto y la Península del Nido, respetando tan sólo, y no completamente, la playa de Las Alcaravaneras.

Una de estas opciones favorecía el desarrollo urbano, era el crecimiento hacia naciente del puerto, que reducía la conflictividad entre los usos más molestos de éste y el uso residencial y turístico de la ciudad. La otra, cerrar el puerto mediante un largo dique con base en el muelle Las Palmas, habría supuesto a día de hoy contar con una ciudad muy distinta a la actual. Sólo la primera de las propuestas llegó a materializarse.

A finales de los años cincuenta el turismo es ya una prioridad en las estrategias económicas del Estado. En este marco, una Orden Ministerial de 12 de noviembre de 1958 creaba las comisiones mixtas entre los Ministerios de la Vivienda y Obras Públicas con la finalidad de estudiar los Planes de Ordenación Urbana que tuviesen incidencia sobre Zona Marítimo Terrestre y evitar en la medida de lo posible que concesiones portuarias aisladas pudieran constituir un impedimento al desarrollo urbano y "poner orden en la anarquía actualmente existente", con especial atención a las playas En 1960 se llegó a redactar el Anteproyecto de aprovechamiento de las playas y zonas marítimoterrestre de la costa que lindan con el núcleo urbano de la ciudad de Las Palmas. En dicho anteproyecto participó la Comisión Provincial de Urbanismo, representada por Manuel de la Peña, el ayuntamiento, a quien representa el arquitecto municipal (Juan Margarit) y el Ingeniero Director del Puerto, Manuel de Granda.

En estos momentos en los que se decidía la relación entre la ciudad y su puerto, hizo entrada en escena un factor externo que jugó un papel importante en el devenir futuro. A nivel nacional, el Gobierno diseñó el Plan de Estabilización Económica o Plan Ullastres (1959), que supuso la formación de grupos de trabajo a nivel local para poner en marcha el que debía ser puente para la apertura

\footnotetext{
${ }^{9}$ Las actas de este grupo de trabajo se pueden consultar en el archivo de la Autoridad Portuaria.
}

de la economía española tras el período de autarquía. Uno de esos grupos de trabajo fue justamente el encargado de analizar el papel de los puertos de la provincia de Las Palmas, coordinado por el propio Ingeniero Director del puerto de aquel entonces, Manuel de Granda 9 . A resultas de este trabajo se llevó a cabo una planificación que tenía por objeto, no sólo el futuro del puerto, sino también de los sectores económicos insulares a los que servía. Considerados éstos, el que se denominó Plan Canarias (en el marco del Plan de Estabilización) realizó una fortísima apuesta por el desarrollo portuario de Las Palmas, proponiendo una dársena exterior que suponía un enorme salto en cuanto al crecimiento portuario.

Al mismo tiempo que se gestaba el Plan Canarias, con una propuesta de crecimiento del puerto hacia naciente, el propio Manuel de Granda redactaba en paralelo un nuevo Plan General de Ordenación del Puerto de La Luz. Como director y responsable, no sólo del puerto, sino del Plan General, Granda hace una apuesta decidida por sus infraestructuras y propone, entre otras iniciativas, un muelle pesquero y astilleros justo en el litoral sur de La Isleta, dentro de la dársena y en contacto con la trama urbana. Durante la fase de información pública fueron muchas las voces que reclamaron alternativas a las propuestas del Plan, viéndose incluso obligado a actuar como moderador el gobernador civil. La contestación ciudadana fue tal que marca de alguna manera un cambio de tendencia en cuanto a la relación del puerto con la ciudad (FIG. 7).

El Plan Granda de 1962 llegó a aprobarse ${ }^{10}$, aunque nunca se materializó pese a diferentes presiones para que se ejecutara. Por el contrario, supuso un cambio al frente de la dirección del puerto y la redacción de un nuevo plan, el de Modesto Vigueras de 1964. Redactado con urgencia en apenas cuatro meses, resulta sin embargo un ejemplo de documento técnico moderno. Recoge las alegaciones emitidas durante la información pública de su antecesor y, sobre todo, contempla las propuestas del Plan Canarias, en especial su alternativa 2, que suponía la creación de un nuevo dique a naciente y, por tanto, la expansión del puerto hacia el este, alejándose de la ciudad. Sería el germen del puerto del siglo XXI.

Sin embargo, y pese a lo dicho, el propio Modesto Vigueras deja aún evidencias del momento de transición en el que nos encontramos y muestras del cambio de enfoque en la redacción de su Plan General, llegando a manifestar en su memoria

\footnotetext{
${ }^{10}$ Aprobado con fecha 28 de junio de 1963 por la Junta de Obras del Puerto que decidió tramitarlo, es decir, elevarlo a la superioridad según escrito de 7-11-1962.
} 


\section{En defensa del porvenir urbanístico de una zona del barrio porteño}

Firmada por numerosas perconallades, pertencientes unas - ia actividades portuarlas J otras a 106 sectores más Eigni-
eleativos de la vida de nuestra eapital. ha afdo presentada al Alcalde do la cludad una instanela para Irnmar la atención corporativa cobre el plan general del Puerto de La Luz expuesto - información pública, ya que el emplazamiento que se pretende asignar a los astilleros de ASVASA y de los varaderos, al Nerte y Bur del muelle de Bants Catalins afectan, segun los firmantes, al futuro urbanistico ce la capital.

- SI en el pasaao-se alce en h Instan $\sqrt{ }{ }^{2}-n u e s t r o s$ antecesores han cometido graves desaclertos en el planeamiento del desarrollo urbanistico, comn to atestiguan los grandes problemas que el crectmiento de la capltal ha creado, guiandose per las convenienelas del momento y por el camino de la menor reslstencla de una parte, y de otra sin una visión clara, amplla de posibles desarrollos futuros, no podemos carrar hoy con la misma responsabllidad para cerrarnes el paso a toda posiblildad de desarrollar en los alrededares del Parque Santa Catallna un cuadro urbanistico digno de una cludad que proximaraente rebasará los 200.000 habitantes", $Y$ sfiade: "La ubleacbu de unos centros indes. triales, por Indis pensables que vean, prechamiente en un silla que puede entar llamado a ser - l lugar de atracelon de nuestra cludad, cuyo centro de rrave. dad te ha tlo desplazando ha. ela el Puerto de Ia Luz, serí on error imperdonable $y$ de costosisima correcelon, cuando not hayamos pereatado de ello. Que if en los momentos presentes quladis no se puede apreclar ein la deblda reflextón, tarde emprano se planteará en tod. ou crudeza, cuando is generaclon que nos sigue, nos acuse de haber desperdlelado una de la pocas posibllidades que nos quedan, por hacer algo para el embelleclmiento de nuestra eapltal. rste centro Industrial que prelende ublear alli, nace con el defecto de toda posible expanalón, como la hace esperar el futuro desarrollo de las actlvi-

dades maritimas $y$ pesqueras $y$ lleva a un lugar de inflnitas pesbilidades creatlvas frente la sineular estampa de la bahia del puerto, el ruíco y las Incomodidades de unos talleres, gee deben quedar alejados del nucleo urbano*.

$$
\text { - (o)- }
$$

Esperamos que la corporacbon municipal intervenga en representación de la eludad y se decida $\mathrm{A}$ acometer en nombre de in misma la necion defensiva que talve ese valineo rineón para otros proyectos que el desarrollo futuro de aquella rona premete y que parece dibujarse en la construcción de la Case det Marino y las edificaciones ce entidindes banchrias en pus stredederes, opontendote a la Intalackion de astilleros y raraderos en los sitios proycetades, para que no ceurra en krande le que de irractadamente no parece tener remedio $y$ que tan radicslmente sfeeta al Parque ce Santa Catalino
F $N$ ESE SECTOR INMIEDIA E to al muelle de Santa Cata. lina está surgtendo una importante industria hotelera que se instalara en el gran edificlo de la Casa del Marino. Ya se ha informado dé que habrá un hotel de unas trescientas habitaciones y otra parte del edilicio estará ciestinada a apartamentos, unes doscientos. Esto obliga a pensar sobre el nuevo emplazamiento que se va a dar a varaderos particulares dentro de esa zona com prendida entre el Muelie de Santa Catalina y la base de hidros. proyecto que se encuentra en exposicion pública para oir reclamaciones y cuyo plazo imaliza. precisamente el lunes dia 25 . I,os varaderos son industrias rutdo sas, con sus molestos martiltos mecánices, etc, y no cabe duda que su Instatacion más apropiada sera lo más distante posibie de nucieos residenciales y hoteleros. Siempre y cuando ello sea compatible con las exigencias primordiales del puerto.

\section{- baIOS rRClaido ta co.}

4 bia de un escrito tirgyter at Alcalde de la ciudad quat at Alcalde de la ciralad que firman cincuenta y cinoo perso. nas repreantativas de otveria actiridades y profestones, con et ruecon de hacernos eco del mismo m MIRADOR. Su texto, del que reproducimos algunos jirrafos, coincide eon lo gue sobre el mismo asunio habiamos expuesto recientemente en exta seccisn.

$$
\text { Af } a
$$

c E PEFIERE EI. ESCRITO al Plan General del Puerto de La Luz expuesto a informacion publica, y llama la atencion sobre un detalle del mismo que - dice - ha de afectar al futuro urbanistico de nuestra ciudad: el emplazamiento que se pretende asignar a los astilleros ie ASVASA y a los varaderos, al Norte y Sur del mutle de Santa Catalina.

FIG. 7/ Extracto de noticias aparecidas en Diario Las Palmas (17/7/1962) y Diario Falange (24/6/1962 y 15/7/62).

Fuente: Diarios y fechas de referencia, consultados en aplicación Jable. ULPGC.

que uno de los condicionantes más importantes es el "aspecto urbanístico y turístico", pues "el turismo ofrece unos incrementos y perspectivas verdaderamente extraordinarias" habiéndose llevado a cabo los crecimientos en torno a las zonas portuarias, en detrimento del propio puerto.
"La realidad es que hoy día existen interferencias entre las necesidades urbanas y las del puerto (...) es necesario ajustar las necesidades del puerto a los deseos de la ciudad",

algo que, pese a la afirmación, Vigueras lo veía como un inconveniente para el puerto, pues era 
anteponer los criterios urbanísticos, turísticos y también militares (en referencia a la base naval), a la que debería ser la expansión portuaria natural, ocupando la franja de litoral hasta el antiguo muelle Las Palmas. Pese a todo ello, proyectó un Plan que, siguiendo las sugerencias de la alternativa 2 del Plan Canarias, supuso el crecimiento del puerto hacia el mar, donde podía emplazar las actividades más molestas. Su materialización tendría lugar por medio del Plan de Ordenación y Obras del Puerto de Argenti Ulloa (1971), quien no hizo más que poner en práctica el Plan Vigueras.

En la memoria del plan de 1971, Argenti Ulloa mencionaba lo siguiente en referencia a su planificación:

"El puerto de La Luz y de Las Palmas, quedará perfectamente acondicionado para la manipulación de mercancías, avituallamiento, pesca, pasajeros y reparación naval. Sin embargo, su proyección como puerto industrial básico está limitada por dos circunstancias fundamentales. Primera, estar rodeado por la ciudad y en una orientación tal, que cualquier industria establecida en e mismo, perturbaría la salubridad del ambiente residencial. En segundo lugar, en la inexistencia de amplias zonas de terreno llano y de poco valor, condiciones indispensables para el asentamiento de los complejos industriales".

El de Argenti Ulloa sería el último de los planes anteriores a la trascendente Ley del Suelo de 1975 (Reglamento de 1976).

\subsection{La etapa "urbanística". Desde 1975 hasta finales del siglo XX}

El cambio de planteamientos respecto a la planificación del espacio portuario se produce entre los años 1975 y 1980 y viene definido de alguna manera por entender el puerto a partir de su interrelación con el espacio confluyente y su entorno, ya no sólo en términos económicos de hinterland o ámbito al que el puerto da servicio, sino como realidad construida y espacio de confluencia, y conflictividad no siempre bien resuelta. En este sentido, el puerto se manifiesta como un polo de atracción realmente potente y con una considerable influencia sobre el desarrollo urbano. Al mismo tiempo, y en especial a raíz de la Ley del Suelo de $1976^{11}$, la planificación comienza a plantearse de manera más integradora y global, de forma que los documentos de ordenación de ambos espacios se influyen y condicionan mutuamente. En cierto sentido, es uno de los objetivos que se plantea la legislación urbanística de 1976: "llenar el vacío existente en el Ordenamiento jurídico en orden a la conexión del planeamiento físico con el planeamiento socio-económico".

A todo ello se suma la incorporación de los aspectos territoriales y ambientales, que pasan a ser considerados en la toma de decisiones a la hora de proceder a construir los espacios urbano y portuario, algo que no había ocurrido hasta ese momento.

Conviene aquí hacer un breve paréntesis aclaratorio. El final del Franquismo y el advenimiento de la democracia y el Estado de las Autonomías supuso un cambio en las competencias territoriales. Sin ánimo de extendernos en demasía sobre este aspecto, que nos desviaría del asunto central, sí que habría que apuntar que el Estatuto de Autonomía de Canarias (Ley Orgánica 10/1982, de 10 de agosto) y todo lo que conlleva el nuevo marco jurídico desde este momento, supuso un nuevo encuadre en la forma de entender la planificación. Esto tendría importantes consecuencias en el enfoque de los instrumentos de ordenación (término que en estos momentos ya nos atrevemos a aplicar, en concordancia con el marco temporal alcanzado).

En relación a la aclaración anterior y siguiendo con el repaso a los planes que nos ha llevado hasta aquí, en el Plan Especial de la zona de servicio del Puerto de la Luz (HeRnández León, 1991), encontramos una evidencia de los nuevos tiempos. En su definición del ámbito del plan dice textualmente:

"Se excluye en la redacción del mismo el resto de la zona de servicio que será objeto de un Plan Especial a desarrollar en colaboración con el Ayuntamiento de Las Palmas",

evidenciando la conjunción entre planeamiento sectorial y urbano. Hernández León alude directamente al PGOU de 1989, que establece:

“(...) coordinar y engarzar con el resto de la ciudad la evolución futura del Puerto, previéndose las necesidades para los distintos tráficos y usos"

(lo que sería función esencialmente portuaria),

"se respetará el borde del litoral junto a la Avenida Marítima para espacios libres, ocio, puerto deportivo, etc. que se desarrollará según otro Plan Especial a redactar entre el ayuntamiento y la Junta de Obras del Puerto".

${ }^{11}$ Real Decreto 1346/1976, de 9 de abril, por el que se aprueba el texto refundido de la Ley sobre Régimen del Suelo y Ordenación Urbana. 


\section{Es lo que Delgado (1998: 246) define como}

\begin{abstract}
"la integración del puerto en la ciudad, provocada por la necesidad de buscar nuevos espacios para el consumo de la actividad comercial propiamente portuaria y la comercial y recreativa que demanda la ciudad".
\end{abstract}

En la misma línea de integración de ambos documentos iría lo expuesto por el Plan Especial del Puerto, en el punto 1.1.4 referida al Objeto del trabajo, donde se lee como tal

"la adecuación armonizada y ligada con los correspondientes terrenos adyacentes del casco urbano de Las Palmas".

Por fin se estudian los desarrollos portuarios tomando en consideración condicionantes urbanísticos (planeamiento físico, si usamos la nomenclatura de la Ley del Suelo), entre los que se mencionan la proximidad de la trama urbana y la existencia de la playa de Las Alcaravaneras, de la que dice no poderse asegurar la compatibilidad de su uso recreativo con los usos portuarios. Incluso es visto como un factor que condiciona el desarrollo portuario la proximidad de la playa de Las Canteras y su desarrollo turístico, que ha "saturado de edificaciones las proximidades del puerto", y también se advierte que el interés del puerto es nacional, "mayor que el particular que sólo puede afectar a un núcleo urbano", lo que debe interpretarse como una reminiscencia de los planteamientos de la etapa que denominábamos "socioeconómica", y un conflicto lógico de intereses entre el puerto y la ciudad. Sin embargo, ya existe un nuevo enfoque al planificar los crecimientos del puerto.

Desde la creación de las autonomías la maquinaria legal en cuanto a la planificación y ordenación se ha vuelto cada vez más compleja, pero se ha ganado en integración entre legislación general y sectorial, adoptándose una visión más territorial. Existe un sistema de planeamiento jerárquico y los documentos que se redactan adquieren una visión global de la que antes carecían.

Por ejemplo, la Ley 48/2003 requiere la aprobación previa de un Plan Director de Infraestructura del Puerto (PDIP) antes de llevar a cabo cualquier ampliación en un puerto del Estado. Este instrumentos será el que defina el modelo de desarrollo y ampliación y cuenta con la potestad de modificar, llegado el caso, un Plan de Utilización de los Espacios Portuarios (PUEP) y, por tanto, puede afectar a la delimitación de la Zona de Servicio que este último plantee ${ }^{12}$. Asimismo, al adquirir Canarias competencias territoriales, será el Gobierno Autonómico el que regule el sistema de planeamiento $y$, aunque sigue estando perfectamente definido el ámbito del Estado dentro de la Zona Marítimo Terrestre y el espacio de competencias regionales o municipales, no obstante, ahora se obliga a la infraestructura portuaria a adoptar un compromiso de integración con su entorno del que antes se carecía.

El ámbito de competencias queda fijado por el deslinde marítimo-terrestre. Sin embargo, y pese a éste, el espacio continúa siendo uno y lo realmente significativo es que, tanto la administración estatal como municipal, resuelvan razonablemente las tensiones territoriales a uno y otro lado de la línea de deslinde, bien por medio de la cesión mediante la fórmula de concesión administrativa, o bien por medio de la desafección (GoNZÁlEZ \& ARMENGOL, 2006). En definitiva, dentro de un nuevo marco competencial y jurídico, se mantiene un interesante diálogo en el espacio de confluencia de la ciudad y el puerto.

\section{Conclusiones}

La ciudad y el puerto han tenido crecimientos y desarrollos paralelos y ambos espacios se han influido mutuamente en este proceso, lo cual creemos que es un hecho constatado. Existe, por tanto, un espacio de correlación entre ambas realidades y éste viene definido por el ámbito de gestión portuaria en confluencia, actualmente, con la ciudad.

Los desarrollos del puerto y la ciudad vienen definidos por los documentos técnicos que diseñan su expansión: proyectos y planes. El estudio de esta documentación permite valorar la evolución acontecida en este ámbito. En este sentido, partíamos de una hipótesis que era el cambio de rol que el puerto ha jugado respecto a la ciudad, y nos planteamos si esta evolución se debía en parte a un cambio de paradigma o enfoque en el proceso de pensar el espacio geográfico portuario, o no. En otras palabras, si el planificador había plasmado en los diferentes documentos sobre los que se produce el crecimiento de la infraestructura portuaria esa forma de entender el puerto en relación a su entorno, en especial a la ciudad.

Se trataba de comprobar algo que se afirmaba como premisa cuando redactamos el Avance del

\footnotetext{
${ }^{12}$ El deslinde de la Zona Marítimo-Terrestre es el límite jurisdiccional de la gestión del Estado, es decir, de Puertos, la gestión municipal (la ciudad).
} 
PGOU de Las Palmas de Gran Canaria (el documento actual aprobado definitivamente en diciembre de 2012) y es que:

"cada uno de los sucesivos Planes Generales (...) está asociado a la etapa concreta en que se elaboraron, con sus características ideológicas, económicas y sociales" (Memoria del documento de Avance del PGOU de 2001, p. 1/29).

El análisis de los planes y proyectos de crecimiento portuario, más incluso que sus equivalentes urbanísticos, han resultado clave en esta investigación. Mediante su estudio ha sido posible establecer una periodización en cuanto a cómo el puerto y la ciudad han entendido el espacio común que les une. Desde unos inicios en los que prevalecían los criterios socioeconómicos (empleamos terminología prestada de la Ley del Suelo de 1975) y el crecimiento del puerto obedecía a razones emitentemente funcionales, hasta una forma de entender éste de manera mucho más global, en la que la planificación se aborda desde una perspectiva más territorial e integradora, donde juega un papel importante la relación del puerto con su entorno y por tanto con la ciudad.

En este sentido, hemos diferenciado lo que denominamos etapa socioeconómica, que abarca hasta la década de 1970 y que incluye dos subetapas, de la etapa "urbanística" que se desarrolla a partir de la promulgación de la Ley del Suelo de 1975 y el casi paralelo desarrollo del Estado de las Autonomías. Entre ambas, hay un período de transición que va desde los planes de comienzo de los años 60 (Plan Canarias, 1962, Plan Granda, 1963 y Plan Vigueras, 1964), hasta el de 1981 (Navarro Miñón) y en los que se observa en los planteamientos de los redactores, y también en la respuesta de los agentes públicos, una búsqueda de soluciones más consensuadas.

En la actualidad, en el diálogo entre ambos espacios parece prevalecer el criterio urbanístico, al menos en el ámbito del litoral más próximo a la ciudad. Las actividades portuarias, especialmente las más agresiva y duras, y por tanto incompatibles con los usos residencial y turístico, han ido siendo relegadas hacia La Isleta, creciendo el puerto hacia naciente. Esto es debido a varios factores: conceptual, en cuanto a la forma de entender la planificación, pero también físico, ya que el crecimiento del puerto se ha encontrado con tres condicionantes muy notables: la limitación de la base naval, que ha cercenado el crecimiento por el sur; la trama urbana, que habiendo crecido al amparo de su puerto y apropiándose de sus terrenos, ha supuesto un freno al crecimiento portuario hacia poniente, y por último, la escasa superficie terrestre disponible, que ha empujado el desarrollo portuario hacia el noreste, ganando terrenos al mar y a La Isleta.

En el marco del proyecto Global South. Puertos y desarrollo económico y social en el Atlántico Meridional, financiado por el MINECO, y dentro por tanto de un estudio mucho más amplio, el análisis de la documentación de los planes de desarrollo del puerto ha permitido secuenciar el cambio territorial y conceptual (en cuanto a la planificación) que se ha producido en el ámbito de estudio: el espacio de interacción del Puerto de La Luz (y de Las Palmas) y la ciudad de Las Palmas de Gran Canaria.

\section{Bibliografía}

Burriel de Orueta, E. (1974): «El puerto de la Luz en Las Palmas de Gran Canaria». CIES, $n^{\circ}$ 18. Las Palmas de Gran Canaria.

Cáceres Morales, E. (2011): «La formación urbana de Las Palmas». Materiales de trabajo, $n^{\circ} 5$. Escuela Técnica Superior de Arquitectura de Las Palmas.

Cirilo Moreno, J. (1947): De los puertos de La Luz y de Las Palmas y otras historias. Ediciones Gabinete Literario. Las Palmas (copia digital accesible en Jable. ULPGC).

Delgado Aguiar, G. (1998): «Las relaciones puertociudad en Las Palmas de Gran Canaria: tensiones y tendencias territoriales». Vegueta, $n^{\circ} 3$; pp. 243-254. Las Palmas de Gran Canaria.

del Nero Beneitez, E. (2007): "Los conflictos competenciales en las intervenciones Puerto-Ciudad. Un ejemplo en Las Palmas de Gran Canaria", Revista de Obras Públicas, septiembre 2007, pp. 19-38. Madrid.

- (2008): "La planificación de los puertos estatales y la ordenación del territorio", Revista de Obras Públicas, septiembre 2008, pp. 39-64. Madrid

González Morales, A. \& Armengol Martin, M.T. (2006): «El Istmo de Santa Catalina en Las Palmas de Gran Canaria: un espacio singular con un controvertido proyecto urbanístico». Vegueta, no 9 pp. 175-192. Las Palmas de Gran Canaria.

Herrera Piqué. A. (1984): Las Palmas de Gran Canaria ( $2^{\mathrm{a}}$ ed.). Editorial Rueda. Madrid. 2 vols.

- (1978): La ciudad de Las Palmas. Noticia histórica de su urbanización. Excmo. Ayuntamiento de Las Palmas de Gran Canaria. Las Palmas de Gran Canaria.

- (2008): "El puerto de la Luz; textos para una exposición conmemorativa", en 125 años de Nuestro Puerto. Una historia unida al desarrollo de la ciudad. Autoridad Portuaria de Las Palmas. Las Palmas de Gran Canaria; pp. 14-64.

Holm Mateo, G. (2013): «El desarrollo del Puerto de Las Palmas a través de la evolución de sus planes». Proyecto fin de Máster. Inédito.

Junta de Obras de los Puertos de la luz y de las Palmas (1928): Memoria. Las Palmas de Gran Canaria.

Martín GalÁn, F. (2001): Las Palmas Ciudad y Puerto. Cinco siglos de evolución. Fundación Puertos de Las Palmas. Madrid. 
- (2007): El mar, la ciudad y el urbanismo. Autoridad Portuaria de Santa Cruz de Tenerife, Autoridad Portuaria de Las Palmas y Fundación Puertos de Las Palmas. Canarias.

Medina SanabriA, J. (1996): Isleta / Puerto de La Luz: Raíces. Autoedición. Las Palmas de Gran Canaria.

Mirallave Izquierdo, V. (1990): Zuazo y Las Palmas. 1940-1968. Tesis Doctoral. Universidad de Las Palmas de Gran Canaria.
SuÁrez BosA. M. (2005): "Cambios en la organización de la actividad en el Puerto de Las Palmas (Gran Canaria): una visión histórica", en Transportes, Servicios y Telecomunicaciones, n. 9 , pp. 66-88.

Tous Melián, J \& Herrera Piqué, A. (1995): Las Palmas dE Gran Canaria a través de la CARTOGRAFía (15881899). Casa de Colón y Museo Militar Regional de Canarias. 